\title{
Maximal Confluent Processes
}

\author{
Xu Wang \\ International Institute of Software Technology, United Nations University \\ PO Box 3058, Macau \\ wx@iist.unu.edu
}

\begin{abstract}
In process semantics of Petri Net, a non-sequential process is a concurrent run of the system represented in a partial order-like structure. For transition systems it is possible to define a similar notion of concurrent run by utilising the idea of confluence. Basically a confluent process is an acyclic confluent transition system that is a partial unfolding of the original system. Given a non-confluent transition system $G$, how to find maximal confluent processes of $G$ is a theoretical problem having many practical applications.

In this paper we propose an unfolding procedure for extracting maximal confluent processes from transition systems. The key technique we utilise in the procedure is the construction of granular configuration structures (i.e. a form of event structures) based on diamond-structure information inside transition systems.
\end{abstract}

\section{Introduction}

Confluence is an important notion of transition systems. Previously there has been extensive work devoted to its study, e.g. [10, 7, 6, 9]. In [7] confluence is studied from the perspective of non-interleaving models, where it was concluded that in order to characterise the class of confluent transition systems the underlying event-based models needs to support the notion of or-causality [16, 19].

In this paper we are going to study the idea of maximal confluent sub-systems of a non-confluent transition system, also from a non-interleaving perspective. It can be regarded as an extension of the notion of non-sequential pocesses in Petri Net $[5,2,3]$ onto transition systems. We call it maximal confluent process (MCP). Intuitively a maximal confluent process is a concurrent run of the system that is maximal both in length and in degree of concurrency. A non-confluent system has multiple such runs. Non-maximal concurrent runs can be deduced from maximal ones, e.g. by restricting concurrency (i.e. strengthening causality relation).

Like non-sequential processes, which can be bundled together to form branching processes of Petri Net, the set of maximal confluent processes (extracted from a given transition system) can coalesce into a MCP branching processes of the original system. Such branching processes record, in addition to causality information, also the 'choice points' of the system at which different runs split from each other. In a non-interleaving setting the 'choice points' are formalised as (immediate) conflicts on events. The arity of the conflicts can be non-binary, 
thus giving rise to the so called finite conflicts. For instance, in state $s 0$ of Figure 1 actions $a, b$ and $c$ form a ternary conflict, which induces the three maximal concurrent runs of the system (i.e. the three subgraphs on the right).

In this paper we propose an unfolding procedure to construct granular configuration structures from transition systems. The procedure preserves the maximality of confluence in such a way that each generated configuration corresponds to a prefix of some maximal concurrent run. Configuration structures are an event structure represented in a global-state based fashion [15, 14]. They support or-causlity as well as finite conflicts.

\section{Motivating Examples}

We first look at two examples in order to build up some intuitions for maximal confluent processes.

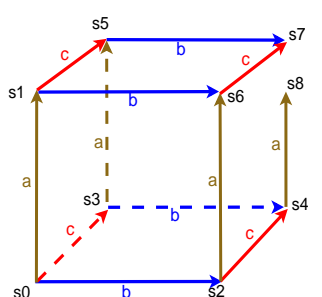

Full Graph

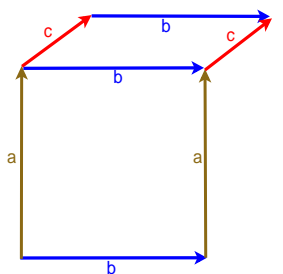

Subgraph 1

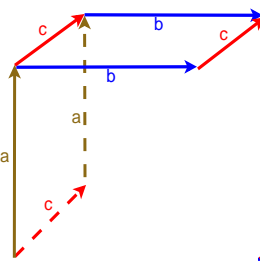

Subgraph 2

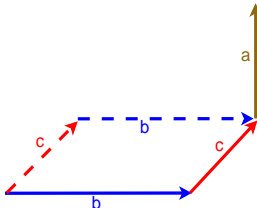

Subgraph 3

Fig. 1. A running example

The first example is the left-most graph in Figure 1, which is an LTS in the shape of a broken cube (i.e. replacing transition $s_{4} \stackrel{s}{\rightarrow} s_{8}$ by $s_{4} \stackrel{s}{\rightarrow} s_{7}$ will give rise to a true cube-shaped LTS). The three subgraphs on its right are confluent subgraphs of the broken cube. Moreover, they are maximal such subgraphs; adding any state or transition to them will invalidate their confluence. They are exactly the maximal confluent processes we are looking for.

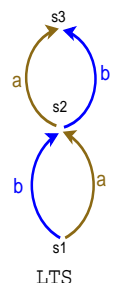

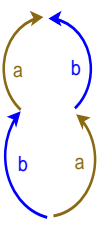

MCP 1

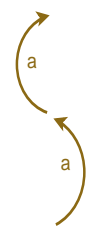

MCP 2

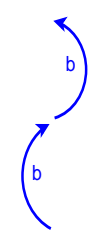

MCP 3

Fig. 2. The second example

For the general cases, however, maximal confluent processes do not coincide with maximal confluent subgraphs. Let us look at the left-most LTS in Figure 2. The maximal confluent subgraphs of such system are the four maximal simple paths in the graph, i.e. $s_{1} \stackrel{a}{\rightarrow} s_{2} \stackrel{a}{\rightarrow} s_{3}, s_{1} \stackrel{a}{\rightarrow} s_{2} \stackrel{b}{\rightarrow} s_{3}, s_{1} \stackrel{b}{\rightarrow} s_{2} \stackrel{a}{\rightarrow} s_{3}$, and 
$s_{1} \stackrel{b}{\rightarrow} s_{2} \stackrel{b}{\rightarrow} s_{3}$. But its maximal confluent processes have three members, MCP 1-3 in Figure 2. Two subgraphs $s_{1} \stackrel{a}{\rightarrow} s_{2} \stackrel{b}{\rightarrow} s_{3}$ and $s_{1} \stackrel{b}{\rightarrow} s_{2} \stackrel{a}{\rightarrow} s_{3}$ are combined into one process, MCP 1 . MCP 1 is not a subgraph because state $s_{2}$ of the original LTS is split into two states.

The idea of maximal confluent processes has interesting applications. The extraction of maximal confluent processes from a given transition system can be regarded as a deep form of commutativity analysis on the system, which is fully dynamic (i.e. state-dependent) and global (i.e. checking infinite number of steps into future). For instance, they can be used in partial order reduction $[11,4,13]$ to define a canonical notion of optimal reduction, weak knot [8]. The challenge, however, lies in how to find a procedure that uses only local diamond-structure information inside transition systems to extract maximal confluent processes.

Now let us develop a formal framework to study the problem.

\section{Maximal Confluent Processes}

Definition 1. A transition system (TS) is a 4-tuple $(S, \Sigma, \Delta, \hat{s})$ where

$-S$ is a set of states,

$-\Sigma$ is a finite set of actions (ranged over by $a, b$, etc.),

$-\Delta$ is a partial function from $S \times \Sigma$ to $S$ (i.e. the transition function) $)^{1}$, and

$-\hat{s} \in S$ is the initial state.

Fix a TS, $G=(S, \Sigma, \Delta, \hat{s})$, and define:

- a transition $t=s \stackrel{a}{\rightarrow} s^{\prime}$ means $\left(s, a, s^{\prime}\right) \in \Delta$;

- a consecutive sequence of transitions $L=s_{0} \stackrel{a_{1}}{\longrightarrow} s_{1} \stackrel{a_{2}}{\longrightarrow} s_{2} \ldots s_{n-1} \stackrel{a_{n}}{\longrightarrow} s_{n}$ means $s_{i-1} \stackrel{a_{i}}{\longrightarrow} s_{i}$ for all $1 \leq i \leq n . L$ is called an execution (i.e. sequential run) of $G$ from $s_{0}$ to $s_{n}$ producing trace $a_{1} \cdots a_{n}$. When $s_{0}=\hat{s}$ we further call it a system execution of $G$; and we use $\mathcal{L}(G)$ to denote the set of system executions of $G$.

$-s \stackrel{a_{1} \cdots a_{n}}{\longrightarrow} s^{\prime}$ means there exists an execution of $G$ from $s_{0}$ to $s_{n}$ producing trace $a_{1} \cdots a_{n}$;

- $s \stackrel{a}{\rightarrow}$ means $\exists s^{\prime}: s \stackrel{a}{\rightarrow} s^{\prime}$, while $s \stackrel{q}{\rightarrow}$ means $\neg s \stackrel{a}{\rightarrow}$;

- $e b(s)$ denotes the set of actions enabled at $s$, i.e. $\{a \mid s \stackrel{a}{\rightarrow}\}$;

- Reach $(s)$ denotes the set of states reachable from $s$;

- given any $s \in \operatorname{Reach}(\hat{s}), G / s=(S, \Sigma, \Delta, s)$ denotes the new transition system generated after the evolution to $s$;

- and if $G$ is acyclic, we further define:

- $s \sqsubseteq s^{\prime}$ means $s^{\prime} \in \operatorname{Reach}(s)$, i.e. $s^{\prime}$ is a subsequent state of $s$ (or $s$ is an earlier state of $\left.s^{\prime}\right)$;

- given any $X \subseteq S, \min (X)$ denotes the set of $\sqsubseteq$-minimal states inside $X$, while $X^{\downarrow}$ denotes the $\sqsubseteq$-downward closure of $X$;

\footnotetext{
${ }^{1}$ Note that our transition systems are actually deterministic transition systems in the classical sense. It gives us simplicity in theory presentation while at the same time sacrificing few technical insights.
} 
- $s \|_{\sqsubset} s^{\prime}$ means $s$ and $s^{\prime}$ are incomparable w.r.t. $\sqsubseteq ;$

- and given any $s \in \operatorname{Reach}(\hat{s}), s / G$ denotes the restriction of $G$ to $\{s\}^{\downarrow}$, i.e. $s / G=\left(\{s\}^{\downarrow}, \Sigma,\left\{\left(s_{0}, a, s_{1}\right) \mid\left(s_{0}, a, s_{1}\right) \in \Delta \wedge s_{0}, s_{1} \in\{s\}^{\downarrow}\right\}, \hat{s}\right)$.

When there is any danger of confusion, we use $\rightarrow_{G}$ to say the transitions come from a TS named $G$. Similarly we use $S_{G}$ for the set of states and $\hat{s}_{G}$ for the initial state of $G$.

TSes can be related to each other by partial unfolding relation:

- We say $G$ is a partial unfolding of $G^{\prime}$ if there exists a function $f$ from $S_{G}$ to $S_{G^{\prime}}$ such that $f\left(\hat{s}_{G}\right)=\hat{s}_{G^{\prime}}$ and $s \stackrel{a}{\rightarrow}_{G} s^{\prime} \Longrightarrow f(s) \stackrel{a}{\rightarrow}_{G^{\prime}} f\left(s^{\prime}\right)$.

As its name suggests partial unfolding unwinds just part of a transition system. When $f$ is injective, partial unfolding is reduced to subgraph relation. In the rest of the paper, whenever the homomorphism $f$ of any subgraph relation is left unspecified, we assume $f$ is the identity function.

Of cause, we can also fully unwind TSes, giving rise to the unfolding relation:

- We say $G$ is an unfolding of $G^{\prime}$ if $G$ is a partial unfolding of $G^{\prime}$ and, for all system execution $\hat{s}_{G^{\prime}}{\stackrel{a_{1}}{\longrightarrow}}_{G^{\prime}} s_{1}^{\prime} \stackrel{a}{\longrightarrow}_{G^{\prime}} s_{2}^{\prime} \ldots s_{n-1}^{\prime}{\stackrel{a_{n}}{\longrightarrow}}_{G^{\prime}} s_{n}^{\prime}$ of $G^{\prime}$, there is a system execution $\hat{s}_{G}{\stackrel{a_{1}}{\longrightarrow}}_{G} s_{1}{\stackrel{a_{2}}{\longrightarrow}}_{G} s_{2} \ldots s_{n-1}{\stackrel{a_{n}}{\longrightarrow}}_{G} s_{n}$ of $G$ s.t. $s_{i}^{\prime}=f\left(s_{i}\right)$ for all $1 \leq i \leq n$.

Of all TSes, a particular interesting subclass of TSes is confluent TSes.

- $G$ is confluent if, for all $s \in S_{G}$ and $a, b \in e b_{G}(s)$ (with $a \neq b$ ), $a$ and $b$ form a local diamond at $s$, i.e. $\exists s_{3} \in S_{G}: s \stackrel{a b}{\longrightarrow}_{G} s_{3} \wedge s \stackrel{b a}{\longrightarrow}_{G} s_{3}$.

In the rest of the paper we use $a \diamond_{s} b$ to denote a diamond rooted at $s$ and built from $a$ and $b$ actions. The notation can be extended to multi-dimension diamonds. We use $\diamond_{s} A$ to denote a $n$-dimension (where $n=|A|$ ) diamond rooted at $s$ and built from members of $A$, i.e. given any $B \subseteq A$, there exists a unique $s^{\prime} \in S$ such that $s \stackrel{a_{1} \cdots a_{m}}{\longrightarrow} s^{\prime}$ for all permutation $a_{1} \cdots a_{m}$ of $B$.

It is interesting to note that all local diamonds inside a partial unfolding are inherited from those of the original TS. They are the unwinded versions of the original diamonds (c.f. MCP 1 in Figure 2). Furthermore, since a partial unfolding can visit a state of the original TS more than once (esp. when the original TS is cyclic), we can choose to unwind a different diamond on subsequent visits to the state.

Now we are ready to define the notion of concurrent runs of TSes:

- We say an acyclic confluent TS $F$ is a confluent process of $G$ if $F$ is a partial unfolding of $G$.

A confluent process $F$ can be finite or infinite. For a finite confluent process $F$, it has a unique maximal state, denoted $\check{s}_{F}$. 
- We say a confluent process $F$ of $G$ is a maximal confluent process (MCPs) if $F$ is maximal w.r.t. partial unfolding relation, i.e. $F$ is a partial unfolding of another confluent process $F^{\prime}$ implies $F^{\prime}$ and $F$ are isomorphic ${ }^{2}$.

When restricted to confluent processes, partial unfolding relation is reduced to subgraph relation (c.f. the lemma below). Thus MCPs are 'maximal confluent subgraphs'. In addition, there is a unique minimal confluent subgraph of $G$, denoted $\hat{G}$. $\hat{G}$ is the trivial TS with a single state and empty transition function, i.e. $\hat{G}=\left(\left\{\hat{s}_{G}\right\}, \Sigma,\{\}, \hat{s}_{G}\right)$.

Lemma 1. Given two confluent processes $F$ and $F^{\prime}$ of $G, F$ is a partial unfolding of $F^{\prime}$ implies the homomorphism $f$ between $F$ and $F^{\prime}$ is injective.

In the rest of the paper we will use $\preceq$ to denote subgraph relation on confluent processes. Relation $\preceq$ allows a confluent process to be reduced in two different dimensions: the degree of concurrency and the length of causality chains. Thus MCPs represent the longest possible runs of the system in a maximally concurrent fashion. In a transition system with cycles that implies MCPs are often infinite graphs: finite MCPs are those derived from terminating runs (i.e. ending in a state where there is no outgoing transitions).

More refined relations on confluent processes that reduces only one of the dimensions can also be defined:

- Given two confluent processes $F$ and $F^{\prime}$, we say $F$ is a (concurrency) tightening of $F^{\prime}$ (or $F^{\prime}$ is a relaxation of $F$ ), denoted $F \preceq_{r} F^{\prime}$, if $F$ is a subgraph of $F^{\prime}$ and, for all $s \in S_{F}$ and $a \in e b_{F^{\prime}}(s)$, there exists a subsequent state $s^{\prime} \in S_{F}$ of $s$ s.t. $a \in e b_{F}\left(s^{\prime}\right)$.

- Given two confluent processes $F$ and $F^{\prime}$ of $G$, we say $F$ is a prefix of $F^{\prime}$ (or $F^{\prime}$ is an elongation of $F^{\prime}$ ), denoted $F \preceq_{e} F^{\prime}$, if $F$ is a subgraph of $F^{\prime}$ and there exists a function $p$ from $S_{F}$ to $2^{\Sigma}$ (i.e. the pending action function) s.t. $s \in S_{F} \Longrightarrow p(s)=e b_{F^{\prime}}(s) \backslash e b_{F}(s)$ and $s \stackrel{a}{\rightarrow}_{F} s^{\prime} \Longrightarrow p(s) \subseteq p\left(s^{\prime}\right)$.

Subgraph relation is decomposable into the two refined relations.

Lemma 2. $F \preceq F^{\prime \prime}$ 1) iff there exists some $F^{\prime}$ s.t. $F \preceq_{r} F^{\prime} \preceq_{e} F^{\prime \prime}$ and 2) iff there exists some $F^{\prime}$ s.t. $F \preceq_{e} F^{\prime} \preceq_{r} F^{\prime \prime}$.

The intuition behind the refined relations can better be understood using the notion of 'events'.

- Given a confluent process $F$, we say a state $s \in S_{F}$ is the origin of an action occurrence, say $a$, if $a \in e b_{F}(s)$ and $s_{0} \stackrel{b}{\rightarrow}_{F} s \Longrightarrow a=b \vee a \notin e b_{F}\left(s_{0}\right)$.

- An occurrence of $a$ with origin $s$ gives rise to a granular event, denoted $T$, which is the set of $a$-transition reachable from $s$ by firing only non- $a$ transitions in $F$. Conversely, given $T$ we use $l b_{F}(T)$ and $o_{F}(T)$ to denote its label $a$ and origin $s$ resp.

\footnotetext{
${ }^{2}$ In the rest of the paper we will freely use $F$ to denote an acyclic confluent graph or to denote its isomorphism class.
} 
- Given two confluent processes $F \preceq F^{\prime}$ and two granular events $T$ in $F$ and $T^{\prime}$ in $F^{\prime}$, we say $T$ and $T^{\prime}$ are the same event if $T \subseteq T^{\prime}$ and $o_{F}(T)=o_{F^{\prime}}\left(T^{\prime}\right)$; and we say $T$ is a postponed occurrence of $T^{\prime}$ if $T \subseteq T^{\prime}$ and $o_{F}(T) \neq o_{F^{\prime}}\left(T^{\prime}\right)$.

- We say two granular events $T$ and $T^{\prime}$ of $F$ are or-causally coupled if $T \cap T^{\prime} \neq$ \{\} .

The or-causal coupling relation is reflexive and symmetric. Its transitive closure, which is an equivalence relation, can be used to partition the set of granular events in $F$. That is, each equivalence class $\mathcal{E}$ gives rise an event $T=\bigcup_{T_{0} \in \mathcal{E}} T_{0}$. Note that an event does not have a unique origin; thus we replace $o_{F}(T)$ by $O_{F}(T)$ to denote its set of origins.

- Given two confluent processes $F \preceq F^{\prime}$ and two events $T$ in $F$ and $T^{\prime}$ in $F^{\prime}$, we say $T$ and $T^{\prime}$ are the same event if $T \subseteq T^{\prime}$ and $O_{F}(T)=O_{F^{\prime}}\left(T^{\prime}\right) \cap S_{F}$, and we say $T$ is a delayed occurrence of $T^{\prime}$ if $T \subseteq T^{\prime}$ and $O_{F}(T) \neq O_{F^{\prime}}\left(T^{\prime}\right) \cap S_{F}$.

Based on the notions of events we can see that tightening on $F^{\prime}$ delays (but not removes) events in $F^{\prime}$ while prefixing on $F^{\prime}$ removes (but not delays) events inside $F^{\prime}$.

One fact noteworthy is that, as we elongate a confluent process, events can become 'enlarged' through the addition of new granular events (even though they remain the same events). However, this addition has an upper-limit as the 'size' of an event will eventually stablise.

Lemma 3. Given a strictly increasing (w.r.t. $\preceq_{E}$ ) infinite sequence of confluent processes $F_{0} F_{1} \ldots F_{i} \ldots, T$ is an event in $F_{i}$ implies there exists some $j \geq i$ and event $T^{\prime}$ in $F_{j}$ s.t. $T$ and $T^{\prime}$ are the same event and $T^{\prime}$ is stablised at $j$, i.e. for any $n \geq j, T^{\prime \prime}$ of $F_{n}$ is the same event as $T^{\prime}$ implies $O_{F_{j}}\left(T^{\prime}\right)=O_{F_{n}}\left(T^{\prime \prime}\right)$.

Some further facts about the refined relations are:

Lemma 4. Given two finite confluent processes $F \preceq F^{\prime}$, we have 1) $F \preceq_{r} F^{\prime}$ iff $\check{s}_{F}=\check{s}_{F^{\prime}}$, and 2) $F \preceq_{e} F^{\prime}$ iff $F=\check{s}_{F} / F^{\prime}$.

In another word the set of prefixes of $F^{\prime}$ corresponds 1-1 to the set of states of $F^{\prime}$.

- A confluent process $F$ is said to be a maximally relaxed process (i.e. MRP) if $F$ is maximal w.r.t. $\preceq_{r}$.

- A confluent process $F$ is said to be an MCP prefix if there exists an MCP $F^{\prime}$ s.t. $F$ is a prefix of $F^{\prime}$. MCP prefixes are the initial parts of complete maximally-concurrent runs.

Naturally one can imagine that MCPs are generated step by step by unfolding local diamonds in the states it visits; MCPs usually prefers to unfold larger diamonds in each step. However, the maximality of MCPs, unlike diamonds, is a global property. Sometimes choosing a strictly smaller diamond to unfold at an early state might lead to a larger diamond in subsequent states. This phenonmenon is similar to the phenonmenon of confusion in Petri Net. 
- Given a state $s \in S_{G}$, we say $A \subseteq \Sigma$ is an MCP step (MPS) at $s$ if there exists a MCP $F$ of $G$ s.t. $\exists s_{F} \in S_{F}: f\left(s_{F}\right)=s \wedge e b_{F}\left(s_{F}\right)=A$.

Given a state $s$, the set of its MPSes are not necessarily downward closed or mutually imcomparable (w.r.t subsethood). As an example, imagine an event structure with four events $e 1, e 2, e 3$ and $e 4$ labelled by action $a, b, c$ and $d$ resp. $e 3$ and $e 4$ causally depend on $e 2$ while $e 3$ is in conflict with $e 1$. In the transition system generated by the event structure, $a$ and $b$ form a maximal diamond at the initial state. However, taking the $a \diamond b$ diamond will destroy the future $c \diamond d$ diamond which is reachable by taking the $b$ action only. Thus $\{a, b\}$ and $\{b\}$ are both MPSes at the initial state whilst $\{a\}$ is not.

Similarly we can see that not all MCP prefixes are MRPs, even though all MRPs are MCP prefixes:

Lemma 5. A confluent process $F$ is an MRP implies $F$ is an MCP prefix.

Maximal confluence is a global property which is generally hard to establish. However, once established, the property is preserved by system evolutions:

Lemma 6. 1) $F$ is an $M C P$ of $G$ implies $F / s_{F}$ is an $M C P$ of $G / f\left(s_{F}\right)$ for all $\hat{s}_{F} \stackrel{a}{\rightarrow}_{F} s_{F}$;2) $\hat{s}_{G} \stackrel{a}{\rightarrow}{ }_{G} s_{G}$ and $F^{\prime}$ is an $M C P$ of $G / s_{G}$ implies there exists an (not necessarily unique) $M C P F$ of $G$ s.t. $\hat{s}_{F} \stackrel{a}{\rightarrow}_{F} s_{F}, s_{G}=f\left(s_{F}\right)$ and $F / s_{F}=F^{\prime}$.

Now we can develop the notion of maximal back-propagation that will form the basis of our unfolding procedure in the next section.

- If $F$ is a confluent process of $G / s$, then we say there is a concurrent run $F$ from $s$, denoted $s \stackrel{F}{\Rightarrow}_{G}$. If $F$ is finite and $f\left(\check{s}_{F}\right)=s^{\prime} \in S_{G}$, we further say that there is a concurrent run $F$ from $s$ to $s^{\prime}$, denoted $s \stackrel{F}{\Rightarrow}_{G} s^{\prime}$.

- We say an action $a \in \Sigma$ is fired in a concurrent run $s \stackrel{F}{\Rightarrow}_{G}$ if an $a$-labelled transition is reachable in $F$. We say an action $a \in \Sigma$ is blocked in a concurrent run $s \stackrel{F}{\Rightarrow}_{G}$ if there exists a path $\hat{s}_{F} \stackrel{a_{1} \cdots a_{n-1}}{\longrightarrow}{ }_{F} s_{F}{\stackrel{a_{n}}{\longrightarrow}}_{F} s_{F}^{\prime}$ in $F$ s.t. $a_{1}, \cdots, a_{n} \in \Sigma \backslash\{a\}$ and $a \diamond_{f\left(s_{F}\right)} a_{n}$ does not hold in $G$; otherwise we say $a$ is unblocked in $s \stackrel{F}{\Rightarrow}_{G}$.

- Given a confluent process $F$ of $G$, we say an action $a \in \Sigma \backslash e b_{F}\left(s_{F}\right)$ is postponed at $s_{F}$ (or, more accurately, the potential granular event with label $a$ and origin $s_{F}$ has postponed occurrence in $F$ ) if $a$ is unblocked in $F / s_{F}$ and there exists a granular event $T^{\prime}$ in $F$ s.t. $l b\left(T^{\prime}\right)=a$ and $o\left(T^{\prime}\right) \sqsupset s_{F}$.

- Furthermore, we say $a \in \Sigma \backslash e b_{F}\left(s_{F}\right)$ is p-pending (partially pending) at $s_{F} \in S_{F}$ if $a$ is unblocked but fired in $F / s_{F}(a)$ and there is no granular event $T^{\prime}$ in $F$ s.t. $l b\left(T^{\prime}\right)=a$ and $o\left(T^{\prime}\right) \sqsupset s_{F}$, and we say $a \in \Sigma \backslash e b_{F}\left(s_{F}\right)$ is pending at $s_{F} \in S_{F}$ if $a$ is neither fired nor blocked in $F / s_{F}(a)$.

For instance, given $G$ (the leftmost graph) and its three confluent processes in the figure below, we can see $c$ is pending at $s 1$, p-pending at $s 2$ and postponed at $s 1^{\prime}$ in the three confluent processes resp. 

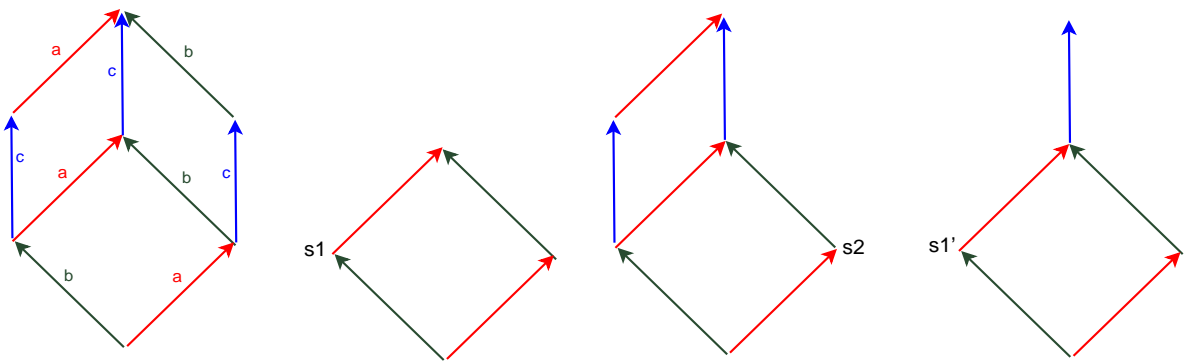

Fig. 3. Pending, p-pending and postponed

- A confluent process $F$ is called primary confluent process (PCP) if no action is postponed at any state in $F$.

- Given a finite confluent process $F$ and an action $a \in e b_{G}\left(f\left(\check{s}_{F}\right)\right)$, we define the pending back-propagation of $a$ over $F$ to be $b p_{p n}(a, F)=\left\{s_{F} \in S_{F} \mid a\right.$ is pending at $\left.s_{F}\right\}$, and the maximal pending back-propagation of $a$ over $F$ to be $\operatorname{mbp}_{p n}(a, F)=\min _{F}\left(b p_{p n}(a, F)\right)$.

- Similarly, given an action $a \in \Sigma$ which is p-pending at some state of $F$, we define the p-pending back-propagation of $a$ over $F$ to be $b p_{p p}(a, F)=\left\{s_{F} \in\right.$ $S_{F} \mid a$ is p-pending at $\left.s_{F}\right\}$, and the maximal p-pending back-propagation of $a$ over $F$ to be $\operatorname{mbp}_{p p}(a, F)=\min _{F}\left(b p_{p p}(a, F)\right)$.

Lemma 7. A confluent process $F$ is an MRP iff there is no postponed or ppending action at any $s_{F} \in S_{F}$.

Lemma 8. Given an action $a \in \Sigma$ postponed or p-pending at a state $s_{F}$ of $F$, there exists a unique minimal relaxation $F^{\prime}$ of $F$, denoted $F^{\prime}=F \uparrow_{s_{F}}^{a}$, s.t. $a \in e b_{F^{\prime}}\left(s_{F}\right)$.

Lemma 9. Given an action $a \in e b_{G}\left(f\left(\check{s}_{F}\right)\right)$ and a state $s_{F} \in b p_{p n}(a, F)$, there exists a unique minimal elongation $F^{\prime}$ of $F$, denoted $F \stackrel{a}{\mathfrak{a}_{s_{F}}} F^{\prime}$, s.t. $a \in e b_{F^{\prime}}\left(s_{F}\right)$.

Theorem 1. Given a finite primary confluent process $F$, if $s_{F} \in m b p_{p p}(a, F) \wedge$ $F^{\prime}=F \uparrow_{s_{F}}^{a}$ or $s_{F} \in m b p_{p n}(a, F) \wedge F \stackrel{a}{\sim_{s_{F}}} F^{\prime}$, then $F^{\prime}$ is a primary confluent process.

\section{Coalescing confluent processes}

A confluent process records one possible history of system evolution. To see other possible evolutions and pinpoint where different evolutions come to deviate and split from each other, we need to coalesce a set of confluent processes into a branching structure. Coalescing operation merges the shared part of evolution histories, and in so doing, makes the 'branching points' explicit.

- Given a set $\mathcal{F}$ of confluent processes of $G$, we use $\operatorname{pr}(\mathcal{F})$ to denote the set of finite prefix of $\mathcal{F}$. Then we can construct a general transition system $G^{\prime 3}$,

\footnotetext{
${ }^{3}$ The reason general transition systems are needed here is largely due to indeterminate evolution covers (introduced below).
} 
called the coalescing of $\mathcal{F}$, s.t. $S_{G^{\prime}}=\operatorname{pr}(\mathcal{F}), \hat{s}_{G^{\prime}}=\hat{G}$, and $F \stackrel{a}{\rightarrow} G_{G^{\prime}} F^{\prime}$ iff $F \preceq_{E} F^{\prime}$ and $\check{s}_{F} \stackrel{a}{\rightarrow}_{F^{\prime}} \check{s}_{F^{\prime}}$. It is crucial to note that, for all $F \in S_{G^{\prime}}, F / G^{\prime}$ is isomorphic to $F$ and, therefore, a confluent process of $G$.

- The notions of granular events can be extended onto $G^{\prime}: t_{1}=F_{1} \stackrel{a}{\rightarrow} G^{\prime} F_{1}^{\prime}$ and $t_{2}=F_{2} \stackrel{a}{\rightarrow} G_{G^{\prime}} F_{2}^{\prime}$ belong to a same granular event in $G^{\prime}$ iff $t_{1}$ belongs to $T_{1}$ in $F_{1}^{\prime} / G^{\prime}, t_{2}$ belongs to $T_{2}$ in $F_{2}^{\prime} / G^{\prime}$ and $\min \left(T_{1}\right)=\min \left(T_{2}\right)$.

Although we can coalesce arbitrary sets of confluent processes, it makes more sense to coalesce a set of confluent processes that are 1) mutually incomparable w.r.t. $\preceq$ and 2 ) able to fully cover the set of system evolutions. The second requirement can be formalised in the same spirit as for the definition of unfolding. A confluent process $F$ covers a set of system executions, i.e. those which are a linearisation of some prefix of $F$, denoted $\operatorname{lin}(\operatorname{pr}(\{F\})) . \mathcal{F}$ fully covers the set of system evolutions if $\mathcal{L}(G)=\operatorname{lin}(\operatorname{pr}(\mathcal{F}))$. We call such set of confluent processes an evolution cover of $G$.

- An evolution cover $\mathcal{F}$ of $G$ is an MCP evolution cover if all $F \in \mathcal{F}$ are MCPs.

- A transition system $G^{\prime}$ is a $C P$ unfolding of $G$ if there exists an evolution cover $\mathcal{F}$ of $G$ s.t. $G^{\prime}$ is the coalescing of $\mathcal{F}$.

- A transition system $G^{\prime}$ is a $M C P$ unfolding of $G$ if there exists an MCP evolution cover $\mathcal{F}$ of $G$ s.t. $G^{\prime}$ is the coalescing of $\mathcal{F}$.

- If, furthermore, for all $L \in \mathcal{L}(G)$, there exists a unique $F \in \operatorname{pr}(\mathcal{F})$ s.t. $L \in \operatorname{lin}(F)$, we call $\mathcal{F}$ determinate.

For determinate evolution covers, we can give a simplified (alternative) definition to CP unfolding:

- We say an acyclic TS $G$ is a confluent tree if, for all $s \in S_{G}, s / G$ is confluent (denoting a concurrent run).

- We say a confluent tree $G^{\prime}$ is a confluent tree unfolding of TS $G$ if $G^{\prime}$ is an unfolding of $G$.

Lemma 10. $G^{\prime}$ is a confluent tree unfolding of $G$ iff there is a determinate evolution cover $\mathcal{F}$ s.t. $G^{\prime}$ is the coalescing of $\mathcal{F}$.

The notion of events on top of confluent tree unfoldings is exactly the same as that on top of confluent processes, i.e. granular events quotiented by an equivalence which is the transitive closure of or-causal coupling relation.

However, the above definition is not extendable to general CP unfolding because of indeterminate evolution cover. For instance, when the two confluent processes in the above figure are coalesced into $G^{\prime}$, transitions $s_{0} \stackrel{b}{\rightarrow} s_{1}$ and $s_{0}^{\prime} \stackrel{b}{\rightarrow} s_{1}^{\prime}$ will collapse in $G^{\prime}$ into one transition, and so are states $s_{2}$ and $s_{2}^{\prime}$ into one state, say $g_{2} \in S_{G^{\prime}}$. (Note that states $s_{3}$ and $s_{3}^{\prime}$ will not collapse in $G^{\prime}$; they are mapped resp. to say $g_{3}, g_{3}^{\prime} \in S_{G^{\prime}}$.) Since $s_{0} \stackrel{b}{\rightarrow} s_{1}$ and $s_{2} \stackrel{b}{\rightarrow} s_{3}$ belong to the same granular event in the left confluent process and $s_{0}^{\prime} \stackrel{b}{\rightarrow} s_{1}^{\prime}$ and $s_{2}^{\prime} \stackrel{b}{\rightarrow} s_{3}^{\prime}$ to the same granular event in the right, using the above definition we can see that 

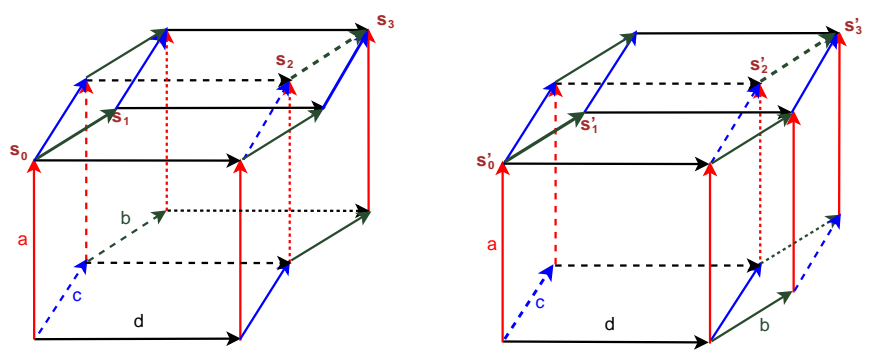

Fig. 4. Label ambiguity v.s. event ambiguity

$s_{2} \stackrel{b}{\rightarrow} s_{3}$ and $s_{2}^{\prime} \stackrel{b}{\rightarrow} s_{3}^{\prime}$ belong to the same event in $G^{\prime}$. Thus, from state $g_{2}$ by firing the same event we can reach two different states, i.e. $g_{3}$ and $g_{3}^{\prime}$. This is contradictory with the intuition of events. In summary, confluent tree unfolding supports the notion of events, whereas CP unfolding only supports the notion of granular events.

So far our problem statement and foundation work are developed mostly within the interleaving framework. But we have witnessed the usefulness of 'event intuition' in understanding notions like prefix, postpone and back-propogation for confluent processes. As we start to deal with more sophisticated CP branching processes, however, we will see that it is crucial (due to simplicity and intuitiveness) to reason directly in terms of events, concurrency, causality and conflict rather than in terms of transitions, commutativity, enabling and disabling.

Thus, we will move gradually into event-based models, e.g. configuration structures and granular configuration structure. Configuration structures are the non-interleaving incarnation of confluent tree unfolding and is thus built from events; while granular configuration structure is the non-interleaving incarnation of CP unfolding and is built from granular events.

Below we start with a quick introduction to the two structures, focusing on the correspondence with their transition system incarnations. Granular configuration structure will be the basis of our MCP unfolding (that requires indeterminate evolution covers, c.f. the example in Figure 1). We will present a formal and detailed introduction of granular configuration structure in the next section ${ }^{4}$.

A configuration structure $(E, C)$ consists of a set $E$ of events and a set $C$ of configurations. Each configuration $c \in C$ is a subset of events, which represents the global state reached after firing exactly the set $c$ of events. The empty configuration represents the initial state.

For example, the left graph in the Figure below is a confluent tree $G$. We can coarsely partition transitions in $G$ into events as shown in the middle graph, or we can finely group them into granular events, which do not form a partitioning of transitions (e.g. $e 3$ and $e 3^{\prime}$ share a transition), as shown in the right graph. In the middle graph each state in $G$ is mapped to a configuration. Given a state $s$ mapped to $c$, if $s$ can transit to $s^{\prime}$ via a transition belonging to $e$, then the $s^{\prime}$ is mapped to $c \cup\{e\}$. The soundness of this rule is implied by the fact that, no

\footnotetext{
${ }^{4}$ A formal and detailed introduction of configuration structures can be found in [8].
} 
matter what system execution one uses to reach a given state in $F$, the set of events fired by the execution is the same.
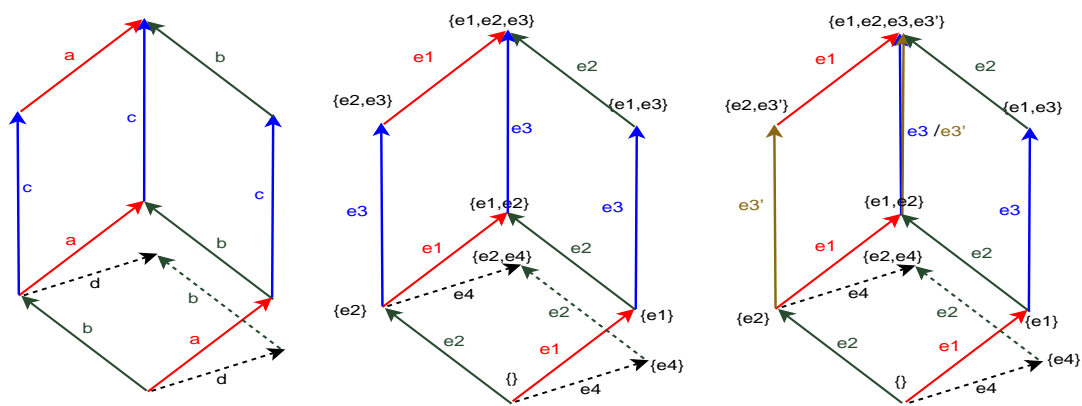

Fig. 5. From transitions to events and granular events

Similarly in the right graph each state $s$ in $G$ is mapped to a granular configuration $c$. But the property that all system executions to a same state fire the same set of events is no longer true. $c$ here denotes, instead, the set of granular events whose member (i.e. transition) has occured in $s / G$. Thus it is possible that, by firing one transition in $G$, we can fire more than one event in the granular configuration structure, e.g. from $\{e 1, e 2\}$ to $\left\{e 1, e 2, e 3, e 3^{\prime}\right\}$ by $c$ and from $\{e 1, e 3\}$ to $\left\{e 1, e 2, e 3, e 3^{\prime}\right\}$ by $b$ in the right graph.

Discussion: The notion of confluent tree unfolding can be of independent interests. Indeed it provides a powerful tool for analysing previous works such as $[18$, $12]$ and [8].

In $[18,12]$ the class of confluent processes one can produce by unfolding a TS $G$ is highly constrained. It is because an independence relation is imposed on top of $G$, i.e. the so called transition systems with independence (TSI). The independence relation marks (statically) a selected subset of diamonds in $G$ as 'true diamonds', and requires all diamonds in confluent processes originating from true diamonds. Furthermore, Axiom 3 of TSI requires that no true diamond can be unfolded sequential, i.e. if two consecutive edges of the diamond are unfolded in $F$, then the whole diamond is unfolded in $F$.

The work in [8] removes the static independence restriction on transitions. Thus a confluent process can utilise any possible diamond in $G$, and a diamond can be unfolded sequentially in one confluent process while concurrently in another one (c.f. the example in Figure 10 of [8]).

Furthermore, Axiom 4 of TSI imposes a transitivity-like condition on the set of true diamonds so that they form a global network of diamonds and the existence of a true diamond at one location implies the existence of a set of true diamonds at its neighboring locations. Therefore, Axiom 4 combined with Axiom 3 ensures that 1) or-causality does not occur in confluent processes (and thus granular events coincide with events), and 2) non-local conditions become reducible to local ones (since the non-local part is guaranteed by the transitivity). 
One example is, given a confluent process $F$ of a TSI $G$ and an event $T$ in $F, T$ is postponed at a state $s \in S_{F}$ that is adjacent to $o(T)$ via transition $s \stackrel{a}{\rightarrow} o(T)$ iff $a \diamond_{f(s)} l b(T)$ in $G$.

Other related works that transform transition systems into non-interleaving models include the region theory of Petri net [1]. However, it is beyond the scope of this paper for detailed comparison.

\section{Unfolding Procedure}

In this section we first introduce granular configuration structures which is an adaptation of labelled configuration structures [15, 14, 8]. Granular configuration structures 1) restore the causality relation on events which can greatly simplify the definition of advanced notions like prefixes, immediate conflicts, etc. and 2) improve the expressiveness so that $\mathrm{CP} / \mathrm{MCP}$ unfoldings can be fully captured. Then we give the $M C P$ unfolding procedure to unfold transition systems into granular configuration structures.

\subsection{Granular configuration structures}

Definition 2. A granular configuration structure (or simply GCS) over alphabet $\Sigma$ is a triple $(E \leq, C, l b)$, where

- E is a partially ordered set of granular events (or henceforth simply events), where $\leq$ is the well-founded causality relation,

- $l b$ is a labelling function mapping events of $E$ into labels of $\Sigma$,

- and $C$ is a set of granular configurations (or simply configurations), where each configuration $c \in C$ is a finite $\leq$-downward closed subset of $E$ and $e \in E$ implies $[e] \in C$.

A configuration $c$ can be thought of as representing a state reached after the execution of exactly the set $c$ of granular events. The empty configuration \{\} represents the initial state and is a required member of $C$ in our model.

Below we fix a GCS, $c s=\left(E^{\leq}, C, l b\right)$, and introduce some basic notions for GCSes.

- We say cs is finitely branching if the Hasse diagram of $E \leq$ is finitely branching. In such a GCS, concurrency and conflict are bounded and infinite configurations are derivable from finite ones.

- $[e]$ denotes the $\leq$-downward closure of $\{e\}$ while $[e]^{-}$denotes $[e] \backslash\{e\}$.

- Given $X \subseteq E,[X]$ and $[X]^{-}$denote $\bigcup_{e \in X}[e]$ and $\bigcup_{e \in X}[e]^{-}$resp.

- We say a nonempty finite subset $\delta \subseteq E$ is a consistent set if there is some $c \in C$ such that $\delta \subseteq c$. Otherwise, $\delta$ is an inconsistent set.

- A consistent $\leq$-downward closed $\delta \subseteq E$ is called a pre-configuration.

- We say an event $e$ is activated at pre-configuration $\delta$, or $e \in a c(\delta)$, if $[e]^{-} \subseteq \delta$ and $c \uplus\{e\}$ is consistent. 
- We say cs is well-activated if $l b(e)=l b\left(e^{\prime}\right) \wedge e \|_{\leq} e^{\prime} \wedge\left\{e, e^{\prime}\right\}$ is consistent implies $[e]^{-} \|_{\subseteq}\left[e^{\prime}\right]^{-}$.

- We say an activated event $e$ at pre-configuration $\delta$ is pending at $\delta$, or $e \in$ $a c_{p n}(\delta)$, if $\forall e^{\prime} \in \delta: e \|_{<} e^{\prime} \Longrightarrow l b(e) \neq l b\left(e^{\prime}\right)$.

- We say an activated event $e$ is p-pending at pre-configuration $\delta$, or $e \in$ $a c_{p p}(\delta)$, if $\exists e^{\prime} \in \delta: e \|_{\leq} e^{\prime} \wedge l b(e)=l b\left(e^{\prime}\right)$. Given a pre-configuration $\delta$, we say $\delta$ is p-pending closed if $a c_{p p}(\delta)=\{\}$; otherwise we use $\delta^{*}$ to denote the p-pending closure of $\delta$.

- We say there is a transition from $c$ to $c^{\prime}$, written $c \stackrel{a}{\rightarrow} C c^{\prime}$, if $c \subset c^{\prime}$ and there exists an event $e \in a c_{p n}(c)$ s.t. $l b(e)=a$ and $\{e\} \subseteq c^{\prime} \backslash c \subseteq(c \uplus\{e\})^{*} \backslash c^{*}$.

The definition of transitions here is unconventional, esp. in comparison with configuration structures. A transition from $c$ to $c^{\prime}$ may involve multiple events $\left(c^{\prime} \backslash c\right)$. Some of them, those pending events from $a c_{p n}(c)$, are the 'driving events' of the transition while others, those p-pending events from $(c \uplus\{e\})^{*} \backslash\left(c^{*} \cup\{e\}\right)$, are 'auxilary ones' piggybacked on the transition. Note that only those freshed generated p-pending events (due to the driving ones) can be piggybacked, not any old one from $c^{*} \backslash c$.

Thus a GCS gives rise to an acyclic transition system, and the definitions like 'subsequent to' relation $\sqsubseteq$, (system) execution, etc. carry over. Furthermore, note that $c \subset c^{\prime}$ does not imply there exists an execution from $c$ to $c^{\prime}$ in GCSes; this is very different from configuration structures.

- We write $e b(c)=\{a \in \Sigma \mid c \stackrel{a}{\rightarrow} C\}$ to denote the set of enabled actions at $c$. $\operatorname{succ}(c)=\left\{c^{\prime} \in C \mid c \stackrel{a}{\rightarrow}_{C} c^{\prime}\right\}$ denotes its set of successor configurations.

- We say $c s$ is well-connected if all the configurations in $C$ are $\rightarrow_{C}$-reachable from \{\} and, for all $c \in C$ and $e \in a c_{p n}(c)$, there exists $c^{\prime} \in C$ s.t. $c \stackrel{l b(e)}{\longrightarrow} C$ $c^{\prime} \wedge e \in c^{\prime}$

Based on the above, we can say cs is well-formed if it is finitely branching, well-activated and well-connected. Well-formed GCSes have roughly the same expressiveness as (general) event structures from [17]. We prefer to use GCSes in this paper mainly because of its affinity to transition systems. We give a few basic properties of well-formed GCSes, esp. those in comparison with configuration structures.

- Given a finite $D \subseteq C$, we say $D$ is downward-closed (w.r.t. $\sqsubseteq$ ) if $c \sqsubseteq c^{\prime} \in$ $D \Longrightarrow c \in D$. We use $D^{\downarrow}$ to denote the $\sqsubseteq$-downward closure of $D$.

- We say a finite subset of configurations $D \subseteq C$ are compatible if $\bigcup D$ is consistent and disjoint from $\bigcup_{c \in D}\left(c^{*} \backslash c\right)$.

- We say cs is closed under bounded union if, for all compatible subsets $D \subseteq C$, $\exists c^{\prime} \in C: \bigcup D \subseteq c^{\prime} \subseteq(\bigcup D)^{*} \backslash \bigcup_{c \in D}\left(c^{*} \backslash c\right)$.

- We say cs is free of auto-concurrency if $l b(e)=l b\left(e^{\prime}\right)$ and $e \|_{\leq} e^{\prime}$ implies $\nexists c \in C:\left[e^{\prime}\right]^{-} \cup[e] \subseteq c \wedge e^{\prime} \in a c(c) .{ }^{5}$

${ }^{5}$ GCSes with auto-concurrency can be useful, on the other hand, for unfolding systems like general Petri Net. 
Lemma 11. cs is free of auto-concurrency and closed under bounded union.

Lemma 12. If there is a non-empty execution from $c$ to $c^{\prime}$ such that $e \in a c(c)$ and $c^{\prime} \cup\{e\}$ is consistent, then we have either $e \in c^{\prime}$ or $e \in \operatorname{ac}\left(c^{\prime}\right)$.

GCSes are the non-interleaving incarnations of the coalescing of confluent processes: each configuration in a GCS uniquely corresponds to an acyclic confluent transition system.

Lemma 13. Given any $c \in C, c s \triangleright\{c\}^{\downarrow}$, i.e. the restriction of cs to $\{c\}^{\downarrow}$, gives

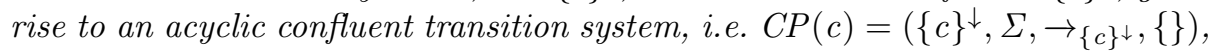
where $C P()$ is an injective function.

For the rest of this paper we only consider well-formed GCSes and simply call them GCSes. Advanced notions of GCSes can be easily defined by using the restored causality relation:

- Given a finite $\leq$-downward closed subset $X \subseteq E$, we say $X$ is $p$-pending event closed (or simply pp-event closed) if, for all pre-configuration $\delta \subseteq X$ and event $e \in X, e \in a c_{p n}(\delta)$ implies $(\delta \cup\{e\})^{*} \subseteq X$. We denote the pp-event closure of $X$ as $X^{\star}$.

- A finite subset $X \subseteq E$ is a prefix if $X$ is both $\leq$-downward closed and pp-event closed.

- A finite $\leq$-antichain $K \subseteq E$ is an immediate conflict (IC) if $[K]^{\star}$ is a minimal prefix that is not conflict-free.

Lemma 14. $K$ is an $I C$ implies $K \subseteq a c_{p n}\left([K]^{-}\right)$.

Based on these notions, we can recover a purely event-based definition of GCS-like structures (i.e. without resorting to the use of configurations):

Granular Event Structures (GESes): A granular configuration structure (say $c s$ ) can be re-formulated (say using transformation $\mathcal{C} \mathcal{E}(c s)$ ) into a granular event structure: a granular event structure is a triple, es $=(E \leq, I C, l b)$, where $I C$ is a set of immediate conflicts (IC). An immediate conflict $K \in I C$ is a finite $\leq$-antichain of events satisfying that, for all $K \in I C,[K]$ contains no IC other than $K$ and, for all $e \in E,[e]$ contains no IC.

Conversely, we can also recover a granular configuration structure from es (say using transformation $\mathcal{E C}(e s)$ ). Given $c s$, we say a finite subset $X \subseteq E$ is consistent if $[X]$ contains no IC. This enables us to recover the definition of pre-configuration, activated/pending/p-pending events and well-activatedness. On well-activated $e s$, we say a pre-configuration $\delta \subseteq E$ is a configuration if there exists another pre-configuration $\delta^{\prime} \supseteq \delta$ s.t. $\left(a c\left(\delta^{\prime}\right) \cup \delta^{\prime}\right) \cap a c_{p p}(\delta)=\{\}$. Finally we say es is well-formed if it is well-activated, finite-branching and satisfying $e \in E \Longrightarrow[e]$ is a configuration.

We can show that well-formed granular event structures correspond exactly to well-formed granular configuration structures:

Theorem 2. $c s=\mathcal{E C}(\mathcal{C} \mathcal{E}(c s))$ and es $=\mathcal{C} \mathcal{E}(\mathcal{E C}(e s))$. 


\subsection{Unfolding TSes into GCSes}

The aim of our procedure is to construct the Hasse diagram of configurations in a roughly bottom up fashion. Starting from the empty configuration, we move up step by step, deriving larger configurations from smaller ones. Each configuration generated corresponds to a finite MCP prefix (up to isomorphism).

However, since transitions between configurations follow 'big-step semantics' (i.e. firing multiple events), a simpler and more elegant approach is to first build the Hasse diagram of pre-configurations, where the transitions follow 'small-step semantics'. Each pre-configuration corresponds to a finite PCP prefix. Then, we remove all pre-configurations that are not configurations (called nonstable preconfigurations) and re-connect what are left, i.e. configurations, by big steps.

The search for new PCP prefixes is guided by a key sub-procedure of extending one PCP (say $F$ ) into another PCP (say $F^{\prime}$ ). Firstly, we calculate the maximal back-propagation of actions for $F$ and, if there is no corresponding events existing for those points, we create new ones accordingly. Then we extend $F$ by those events to generate $F^{\prime}$ (i.e. elongate or relax $F$ depending on the events being pending or p-pending), which is a PCP according to Theorem 1. Note that in generating $F^{\prime}$ there is a 'prefix-closure' effect. That is, $F^{\prime}$ might have more than one immediate prefix and some of them (other than $F$ ) might be non-PCP and, therefore, not generated yet. Thus in generating $F^{\prime}$ we also need to generate some of its prefixes.

Now let us formalise the unfolding procedure and define the notions of $M C P$ unfolding and MCP branching processes:

- Given a TS $G$, a labelled $G C S$ over $G$ is a tuple $l c s=\left(E^{\leq}, C, l b, s t\right)$, where $\left(E^{\leq}, C, l b\right)$ constitutes a GCS over $\Sigma$ and $s t: C \rightarrow S_{G}$ is a function mapping configurations to states.

- Given lcs over $G$, we say lcs is an MCP unfolding of $G$ if $\left(C, \Sigma, \rightarrow_{C},\{\}\right)$ is an MCP unfolding of $G$ via homomorphism $f=s t$.

- Given lcs over $G$, we say $l c s=(E \leq, C, l b, s t)$ is an $M C P$ branching process of $G$ if there exists an $M C P$ unfolding $l c s^{\prime}=\left(E^{\prime \leq}, C^{\prime}, l b^{\prime}, s t^{\prime}\right)$ of $G$ s.t. $E$ is a prefix of $\left(E^{\prime \leq}, C^{\prime}, l b^{\prime}\right), C=C^{\prime} \triangleright E, l b=l b^{\prime} \triangleright E$ and $s t=s t^{\prime} \triangleright C$.

Our procedure, lcs $=\mathcal{U}(G)$ (c.f. Figure 6), unfolds TSes in a maximally concurrent fashion into a labelled GCS over $G$.

The basic data structure is $(E, p r e C, l b, s t) . E$ and preC store resp. the set of generated events and the set of generated pre-configurations. The backpropagation information for each pre-configuration is stored in function $b p_{p n}$ and $b p_{p p}$ resp. We create new events based on such information, which are then passed on from smaller pre-configurations to larger ones and stored in function $a c_{p n}$ and $a c_{p p}$ resp. as activated events.

Then, adding activated events to existing pre-configurations produces the set of potential extensions, EXT. Some of the extensions are PCPs, i.e. those in the set PCP. The definition of PCP is recursive, utilising Theorem 1 and starting from the empty pre-configuration. But, due to the 'prefix closure' effect mentioned above, PCP extensions cannot be realised 'eagerly' by immediately throwing 
them into preC. Rather, the realisation proceeds in steps by first realising the prefixes (i.e. sub-configuration ${ }^{6}$ ) of the PCP extensions, so that the expansion of preC will preserve the $\preceq$-downward closedness. We say a PCP prefix is ready for realisation if it is unrealised but all its sub-configurations are realised. The set of ready-for-realisation PCP prefixes is given in NXT.

Thus, starting from an empty pre $C$ we pick one pre-configuration (say $c^{\prime}$ ) a time from NXT and realise by adding $c^{\prime}$ to preC (line 3-5 of function UNFOLD in Figure 6). In the mean time we calculate (c.f. line 8-9 of function REALISE) the set of activated events inheritable by $c^{\prime}$ from its immediate sub-configurations (i.e. ${ }^{\bullet} c^{\prime}$ ), and also derive (line 4-7 of function REalise) the new $b p_{p n}$ and $b p_{p p}$ functions based on those of $\boldsymbol{c}^{\prime}$. Some useful notations used in such calculation/derivation are given below.

Given any $c, c^{\prime}=c \uplus\{e\} \in$ preC, we define

$$
\begin{aligned}
& -\operatorname{prp}_{p n}\left(c^{\prime}, c, a\right)= \\
& \text { - }\{[e]\} \text { if } e \in a c_{p n}(c) \wedge \neg l b(e) \diamond_{s t(c)} a \vee e \in a c_{p p}(c) \wedge m b p_{p n}(c, a)=\{c\} \text {, or } \\
& \text { - } \min \left(\operatorname{mbp}_{p n}(c, a) \cup\{[e]\}\right) \text { if otherwise; } \\
& -\operatorname{prp}_{p p}\left(c^{\prime}, c, a\right)= \\
& \text { - } b p_{p p}(c, a) \text { if } l b(e) \neq a \text {, } \\
& \text { - }\left\{c_{0} \in b p_{p p}(c, a) \mid c_{0} \|_{\sqsubset}[e]^{-}\right\} \text {if } e \in a c_{p p}(c) \wedge l b(e)=a \text {, or } \\
& \text { - }\left\{c_{0} \in b p_{p n}(c, a) \cup b p_{p p}(c, a) \mid c_{0} \|_{\sqsubseteq}[e]^{-}\right\} \text {if } e \in a c_{p n}(c) \wedge l b(e)=a \text {; } \\
& -h t_{p n}\left(c^{\prime}, c\right)=\left\{e^{\prime} \in a c_{p n}(c) \mid e \in a c_{p p}(c) \vee\left(e \in a c_{p n}(c) \wedge l b(e) \diamond_{s t(c)} l b\left(e^{\prime}\right)\right)\right\} \\
& -h_{t} t_{p p}\left(c^{\prime}, c\right)=\left\{e^{\prime} \in\left(a c_{p n}(c) \cup a c_{p p}(c)\right) \mid\left(e^{\prime} \in a c_{p n}(c) \Longrightarrow e \in a c_{p n}(c) \wedge\right.\right. \\
& \left.\left.l b(e)=l b\left(e^{\prime}\right)\right) \wedge\left(l b(e)=l b\left(e^{\prime}\right) \Longrightarrow\left[e^{\prime}\right]^{-} \|_{\sqsubseteq}[e]^{-}\right)\right\} \text {. }
\end{aligned}
$$

Note that $h r t_{p n}\left(c^{\prime}, c\right)$ produces the set of pending events $c^{\prime}$ can inherit from $c$ while $\operatorname{hrt}_{p p}\left(c^{\prime}, c\right)$ produces the set of p-pending events $c^{\prime}$ can inherit from $c$. On the other hand, $\operatorname{prp}_{p n}\left(c^{\prime}, c, a\right)$ produces the information about how action $a$ can be back-propagated through edge $\left(c, c^{\prime}\right)$ into the sub-configurations while $\operatorname{prp}_{p p}\left(c^{\prime}, c, a\right)$ about the p-pending points of $a$ through edge $\left(c, c^{\prime}\right)$.

Then, if $c^{\prime}$ is a PCP and has maximal back-propagation not covered by activated events inherited from ${ }^{\bullet} c^{\prime}$, we create new ones to cover them (c.f. procedure GenEvent). An event (say e) can be created only if its origin (say $c$ ) is a configuration according to $c^{\prime}$ : the condition is implemented as the predicate $c$ ISCFGIN $c^{\prime}$. After $e$ is created at $c$, we propagate $e$ upward to its super-configurations (c.f. line 4-8 of procedure GenEvent).

Finally, after the set of pre-configurations fully generated, we filter out nonstable pre-configurations and produce the set of configurations (c.f. line 6 of function UNFOLD). The intuition is that a PCP pre-configuration is a MRP if $a c_{p p}\left(c^{\prime}\right)=\{\}$ and the prefixes of MRP pre-configurations are configurations.

We can illustrate the procedure by unfolding the broken cube originally from Figure 1. In the step 0 of Figure 7 the initial state is mapped to configuration \{\} . The set of activated events at \{\} , i.e. $a c_{p n}(\{\})$ and $a c_{p p}(\{\})$, is initialised

\footnotetext{
${ }^{6}$ Actually it should be sub-pre-configurations, i.e. pre-configurations which are subset of the original pre-configurations.
} 


\section{Data Structure:}

$$
\begin{aligned}
& (E, \text { preC }, l b, s t)=(\{\},\{\{\}\},\{\},\{(\{\}, \hat{s})\}) ; \\
& a c_{p n}=\{\} ; a c_{p p}=\{\} ; b p_{p n}=\{\} ; b p_{p p}=\{\} ;
\end{aligned}
$$

\section{Derived Values, Functions and Predicates:}

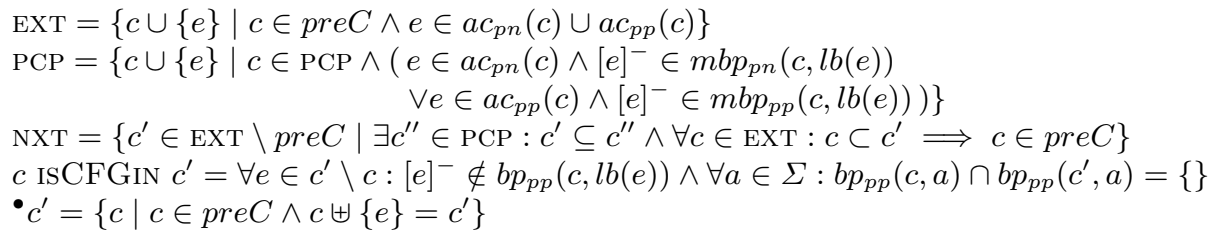

Function $\operatorname{Unfold}((S, \Sigma, \Delta, \hat{s}))$

\section{Begin}

1 Foreach $a \in e b(\hat{s})$ do Set $b p_{p n}(\{\}, a)=\{\{\}\}$;

$2 \operatorname{GenEvent}(\{\})$;

While $\mathrm{NXT} \neq\{\}$

Pick any $c^{\prime} \in$ NXT and Realise $\left(c^{\prime}\right)$;

If $c^{\prime} \in \mathrm{PCP}$ Then GenEvent $\left(c^{\prime}\right)$;

Set $C=\left\{c \in\right.$ pre $\left.C \mid \exists c^{\prime} \in \mathrm{PCP}: c \subseteq c^{\prime} \wedge a c_{p p}(c) \cap c^{\prime}=\{\}=a c_{p p}\left(c^{\prime}\right)\right\}$;

7 Return $(E, C, l b, s t)$

\section{End}

\section{Procedure Realise $\left(c^{\prime}\right)$}

Begin

1 Assume $c^{\prime}=c \uplus\{e\}$ for some $c \in$ preC;

2 If $e \in a c_{p n}(c)$ Then $s^{\prime}=\Delta(s t(c), l b(e))$ Else $s^{\prime}=s t(c)$;

3 Add $c^{\prime}$ to pre $C$ and $\left(c^{\prime}, s^{\prime}\right)$ to $s t()$;

4 Foreach $a \in e b\left(s t\left(c^{\prime}\right)\right)$ do Set $b p_{p n}\left(c^{\prime}, a\right)=\bigcap_{c \in c^{\prime}}\left\{c_{1}: \operatorname{pre} C \mid \exists c_{0} \in \operatorname{prp}_{p n}\left(c^{\prime}, c, a\right): c_{0} \subseteq c_{1} \subseteq c^{\prime}\right\}$;

Foreach $a \in \Sigma$ s.t. $D=\bigcup_{c \in \bullet^{\prime}} \operatorname{prp}_{p p}\left(c^{\prime}, c, a\right) \neq\{\}$ do Set $b p_{p p}\left(c^{\prime}, a\right)=\left\{c_{0} \in D \mid \forall c \in c^{\prime}: c_{0} \subseteq c \Longrightarrow c_{0} \in \operatorname{prp}_{p p}\left(c^{\prime}, c, a\right)\right\}$;

8 Set $a c_{p n}\left(c^{\prime}\right)=\left\{e^{\prime} \in \bigcup_{c \in \bullet_{c^{\prime}}} \operatorname{hrt}_{p n}\left(c^{\prime}, c\right) \mid \forall c \in{ }^{\bullet} c^{\prime}:\left[e^{\prime}\right]^{-} \subseteq c \Longrightarrow e^{\prime} \in \operatorname{hrt}_{p n}\left(c^{\prime}, c\right)\right\}$;

9 Set $a c_{p p}\left(c^{\prime}\right)=\left\{e^{\prime} \in \bigcup_{c \in c^{\prime}} h t_{p p}\left(c^{\prime}, c\right) \mid \forall c \in c^{\bullet} c^{\prime}:\left[e^{\prime}\right]^{-} \subseteq c \Longrightarrow e^{\prime} \in \operatorname{hrt}_{p p}\left(c^{\prime}, c\right)\right\}$

End

\section{Procedure GenEvent $\left(c^{\prime}\right)$}

\section{Begin}

1 Foreach $(a, c) \in m b p_{p p}\left(c^{\prime}\right) \cup m b p_{p n}\left(c^{\prime}\right)$ s.t. $\nexists e \in E:\left(l b(e),[e]^{-}\right)=(a, c) \wedge c$ ISCFGIN $c^{\prime}$ do

Create a new event $e$, and add $e$ to $E$ and $(e, a)$ to $l b()$;

Add $e$ to $a c_{p n}(c)$ and set $D=\{c\}$;

While $M=\min (\{x \in \operatorname{pre} C \mid x \supset c\} \backslash D) \neq\{\}$ do

Pick any $c^{\prime \prime} \in M$ and add $c^{\prime \prime}$ to $D$;

If $\forall c \in{ }^{\prime \prime} c^{\prime \prime}:[e]^{-} \subseteq c \Longrightarrow e \in h r t_{p n}\left(c^{\prime \prime}, c\right)$ Then Add $e$ to $a c_{p n}\left(c^{\prime \prime}\right)$

Else If $\forall c \in{ }^{\prime \prime} c^{\prime \prime}:[e]^{-} \subseteq c \Longrightarrow e \in h r t_{p p}\left(c^{\prime \prime}, c\right)$

Then Add $e$ to $a c_{p p}\left(c^{\prime \prime}\right)$ Else Add $\left\{c \in\right.$ pre $\left.C \mid c \supseteq c^{\prime \prime}\right\}$ to $D$

Fig. 6. An unfolding procedure 
to be empty (i.e. no inheritance). Since the three enabled actions at the initial state are maximally back-propagated to \{\} but there is no activated events at \{\} to match them, we create three new events $e 1, e 2$ and $e 3$ at \{\} (note the use of symbol !) and add them to $a c_{p n}(\{\})$. Thus the initial state is labelled as \{\}$/\{e 1, e 2, e 3\}$ (in the style of $\left.c / a c_{p n}(c)\right)$. Firing one of the generated events say $e 1$ leads to a new extension, say $\{e 1\}$, which is a new member of NXT.

In the step 1 , we pick a member say $\{e 1\}$ of NXT and realise it. $\{e 1\}$ is mapped to $s_{1}$ and $a c_{p n}(\{e 1\})$ inherits $\{e 2, e 3\}$ from \{\} , which fully covers the maximal back-propagation of the two actions enabled at $s_{1}$. Thus, although $\{e 1\}$ is a PCP and procedure GEnEvent is called, no new event will be created. Similarly we can also realise $\{e 2\}$ and $\{e 3\}$.

Now $\{e 1, e 3\}$ is a member of NXT, which we can pick in the step 2 to realise. Note that $\{e 1, e 3\}$ can inherit $e 2$ from $\{e 1\}$ but not from $\{e 3\}$ (since $e 2$ and $e 1$ do not form a diamond at $\{e 3\})$. This inconsistency leads to $e 2$ not being added to $a c_{p n}(\{e 1, e 3\}) .\{e 1, e 3\}$ is a PCP and in calling GEnEvEnT, however, a new event $e 2^{\prime}$ is created at $\{e 1\}$ to cover the maximal back-propagation of the enabled $b$ action at $s_{5}$ (mapped to $\left.\{e 1, e 3\}\right) . e 2^{\prime}$ can be inherited and added to $a c_{p n}(\{e 1, e 3\})$. Similarly, the back-propagation from $\{e 1, e 2\}$ and from $\{e 2, e 3\}$ leads to the creation of $e 3^{\prime}$ at $\{e 1\}$ and $e 1^{\prime}$ at $\{e 2, e 3\}$ resp.

$e 1^{\prime}$ at $\{e 2, e 3\}$ can lead to a PCP extension but not so for $e 2^{\prime}$ and $e 3^{\prime}$ at $\{e 1\}$. Instead, they are PCP prefixes since $\left\{e 1, e 2, e 3^{\prime}\right\}$ and $\left\{e 1, e 2^{\prime}, e 3\right\}$ are PCP extensions. Therefore, all the possible extensions so far are inside NXT. In realising these extensions, $\left\{e 1, e 3^{\prime}\right\}$ and $\left\{e 1, e 2^{\prime}\right\}$ need to be realised before $\left\{e 1, e 2, e 3^{\prime}\right\}$ and $\left\{e 1, e 2^{\prime}, e 3\right\}$ resp. to preserve the $\preceq$-downward closedness of pre $C$.

In the step 3 , obviously $\left\{e 1, e 2^{\prime}\right\}$ inherits $e 3$ and $e 3^{\prime}$ from $\{e 1\}$; and $\left\{e 1, e 3^{\prime}\right\}$ inherits $e 2$ and $e 2^{\prime}$. But $e 3^{\prime}$ at $\left\{e 1, e 2^{\prime}\right\}$ and $e 2^{\prime}$ at $\left\{e 1, e 3^{\prime}\right\}$ will lead to extensions outside NXT. So the outcome is a GCS with three maximal configurations. Two of them, $\left\{e 1, e 3, e 2^{\prime}\right\}$ and $\left\{e 1, e 2, e 3^{\prime}\right\}$, are mapped to a same terminating state $s 7$. (In contrast, state $s 5$ is split into $\{e 1, e 3\}$ and $\left\{e 1, e 3^{\prime}\right\}$ while $s 6$ into $\{e 1, e 2\}$ and $\left\{e 1, e 2^{\prime}\right\}$.)

The broken cube example does not have or-causality; thus the part of the procedure related to non-stable pre-configuration and p-pending points/events has not been utilised. We give a second example in Figure 8 to do so.

The original transition system is given as the top-left graph in the figure. The top-right graph is its MCP unfolding, which is the coalescing of two MCPs $(\{a 1, b 1, c 1, c 2, d 2\}$ and $\{a 1, b 1, c 2, d 1, d 2\})$. The MCP of $\{a 1, b 1, c 1, c 2, d 2\}$ is drawn with thick-line edges and strong-colored configurations.

The bottom graph is the Hasse diagram of pre-configurations (with some edges missing). The faint-colored pre-configurations in bottom graph are nonstable pre-configurations; they will be removed in order to produce the Hasse diagram of configurations. Note further that $\{a 1, b 1, c 2\}$ is a configuration in the MCP of $\{a 1, b 1, c 1, c 2, d 2\}$ but not so in that of $\{a 1, b 1, c 2, d 1, d 2\}$, even though it is a pre-configuration being used in the generation of both MCPs. Note that the set of pre-configurations being used to generate the MCP of $\{a 1, b 1, c 1, c 2, d 2\}$ are those connected up by edges in the graph. 

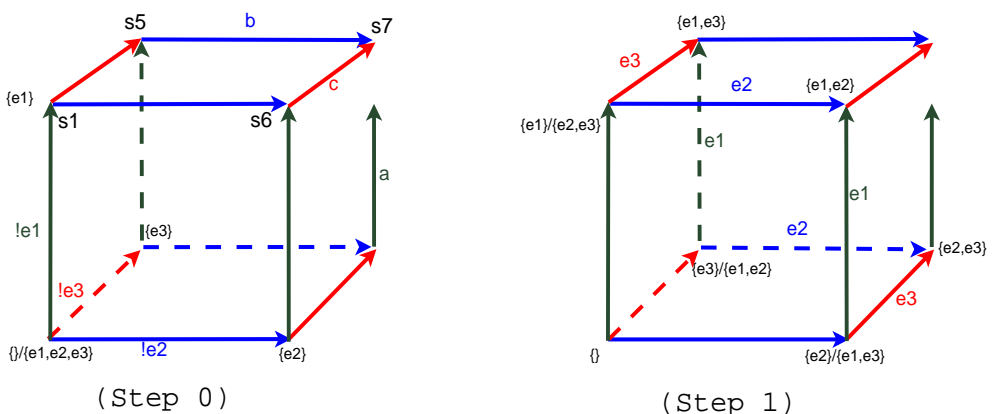

(Step 1)

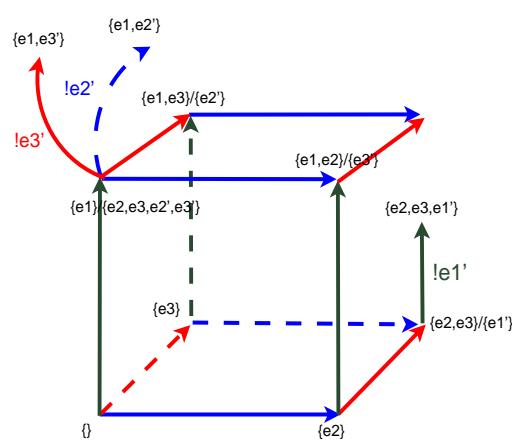

(Step 2)

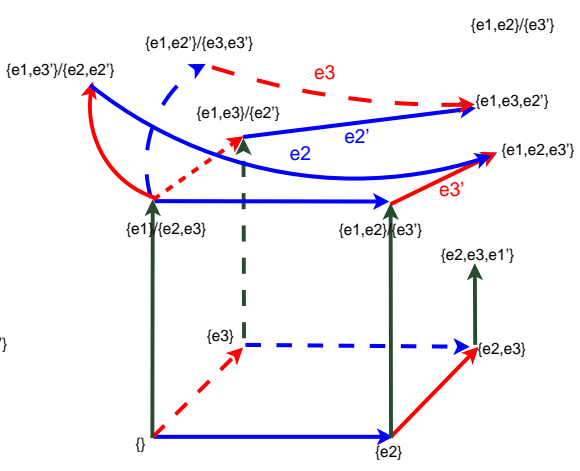

(Step 3)

Fig. 7. Unfolding of the broken cube
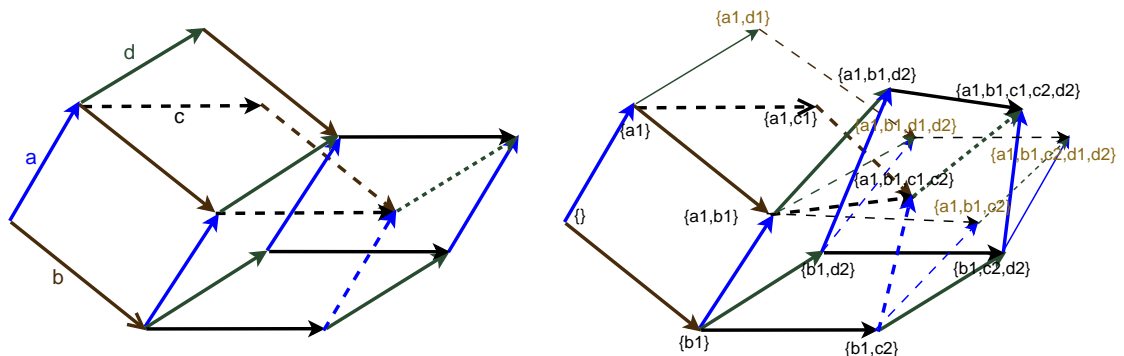

$\{a 1, d 1\} \quad\{a 1, b 1, d 2\} \quad\{a 1, b 1, d 1, d 2\}$

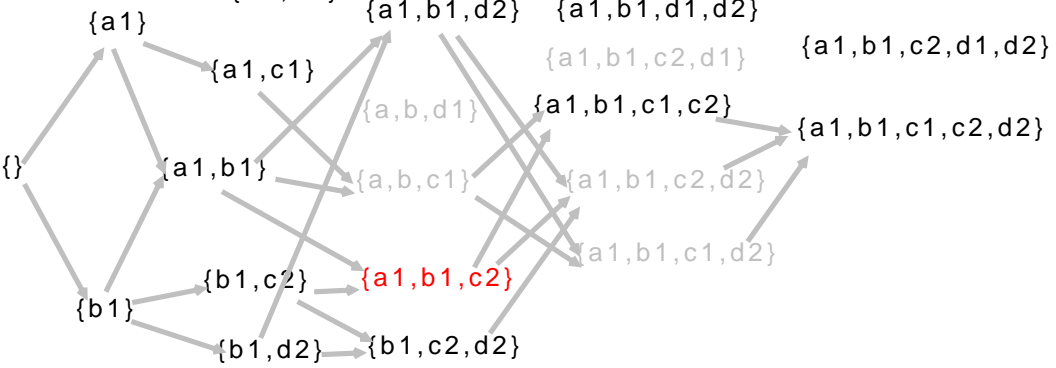

Fig. 8. P-pending event and nonstable pre-configuration 
We can show that the output of function UNFOLD indeed is the MCP unfolding of $G$ :

Theorem 3. $\mathcal{U}(G)$ is an $M C P$ unfolding of $G$.

Acknowledgements We thank Xiaoquan Wu for implementing a prototype unfolder based on the algorithm of this paper. Bill Roscoe and Henri Hansen commented on the earlier ideas of the paper. We acknowledge support from the Macau FDCT project 041/2007/A3 and EU FP7 project IST 231167.

\section{References}

1. Eric Badouel, Luca Bernardinello, and Philippe Darondeau. The synthesis problem for elementary net systems is np-complete. Theor. Comput. Sci., 186(1-2):107-134, 1997.

2. Eike Best and Raymond R. Devillers. Sequential and concurrent behaviour in petri net theory. Theor. Comput. Sci., 55(1):87-136, 1987.

3. Eike Best and César Fernández. Nonsequential processes : a Petri net view, volume 13 of EATCS Monographs on TCS. Springer-Verlag, 1988.

4. P. Godefroid. Partial-order Methods for the Verification of Concurrent Systems: an Approach to the State-explosion Problem, volume 1032 of LNCS. Springer, 1996.

5. Ursula Goltz and Wolfgang Reisig. The non-sequential behavior of petri nets. Information and Control, 57(2/3):125-147, 1983.

6. J.F. Groote and M. P. A. Sellink. Confluence for process verification. Theoretical computer science, 170(1-2):47-81, 1996.

7. Jeremy Gunawardena. Causal automata. TCS, 101(2):265-288, 1992.

8. Henri Hansen and Xu Wang. On the origin of events: branching cells as stubborn sets. In PETRI NETS 2011, LNCS 6709, 2011.

9. Xinxin Liu and David Walker. Partial confluence of proceses and systems of objects. TCS, 206(1-2):127-162, 1998.

10. Robin Milner. Concurrency and Communication. Prentice-Hall, 1988.

11. D. A. Peled. All from one, one for all: on model checking using representatives. In Proceedings of the 5th International Conference on Computer Aided Verification, volume 697 of $L N C S$, pages 409-423. Springer, 1993.

12. Vladimiro Sassone, Mogens Nielsen, and Glynn Winskel. Models for concurrency: Towards a classification. Theor. Comput. Sci., 170(1-2):297-348, 1996.

13. A. Valmari. Stubborn sets for reduced state space generation. In ICATPN, volume 483 of $L N C S$, pages 491-515. Springer, 1989.

14. R. J. van Glabbeek and G. D. Plotkin. Configuration structures, event structures and petri nets. Theoretical Computer Science, 410(41):4111-4159, 2009.

15. Rob van Glabbeek and Ursula Goltz. Refinement of actions and equivalence notions for concurrent systems. Acta Informatica, 37(4):229-327, 2001.

16. Rob J. van Glabbeek and Gordon D. Plotkin. Event structures for resolvable conflict. In MFCS 2004, volume 3153 of LNCS, pages 550-561, 2004.

17. Glynn Winskel. Event structures. In Advances in Petri Nets, volume 255 of LNCS, pages 325-392. Springer, 1987.

18. Glynn Winskel and Mogens Nielsen. Models for concurrency. In Handbook of logic in Computer Science, volume 4. Clarendon Press, 1995.

19. A. Yakovlev, M. Kishinevsky, A. Kondratyev, L. Lavagno, and M. PietkiewiczKoutny. On the models for asynchronous circuit behaviour with or causality. FMSD, 9(3):189-233, 1996. 